\title{
Periprosthetic joint infection with streptococcus dysgalactiae subspecies equisimilis: Case report
}

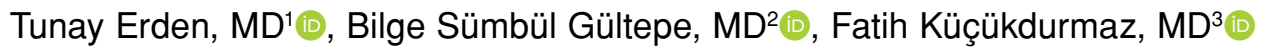 \\ 'Department of Orthopedics and Traumatology, Acıbadem Fulya Hospital, Istanbul, Turkey \\ 2Department of Clinical Microbiology, Bezmialem Vakıf University Medical School, Istanbul, Turkey \\ ${ }^{3}$ Department of Orthopedics and Traumatology, Marmara University, Faculty of Medicine, Istanbul, Turkey
}

Human streptococci that belong to Streptococcus dysgalactiae (SD) have long been known as nonpathogenic for many years, but are now recognized as an important bacterial pathogen. They are classified under the name of beta-hemolytic groups $C$ and $G$ streptococci. ${ }^{[1]}$

Streptococcus dysgalactiae infections are mostly seen after animal contact or associated with the consumption of unpasteurized dairy food products. There are two subspecies of SD, subspecies dysgalactia (SDSD, an animal pathogen) and subspecies equisimilis (SDSE, a human pathogen). Streptococcus dysgalactiae subspecies equisimilis is a group $C$ streptococci and primarily presents as skin and soft-tissue infections, including pyoderma, cellulitis, wound infections, abscesses, erysipelas, and necrotizing fasciitis. ${ }^{[2-5]}$

According to our knowledge, there is only one case report identifying SDSD in a periprosthetic joint infection (PJI), while there is no PJI case reported that is infected with SDSE in the English literature. ${ }^{[6]}$

\section{CASE REPORT}

A 77-year-old retired male patient referred to our clinic with complaint of swelling and redness in his

Received: November 12, 2019

Accepted: January 28, 2020

Published online: June 18, 2020

Correspondence: Tunay Erden, MD. Acıbadem Fulya Hastanesi Ortopedi ve Travmatoloji Bölümü, 34349 Beşiktaş, istanbul, Türkiye.

E-mail: doktorerden@hotmail.com

Doi: $10.5606 /$ ehc. 2020.73840

Citation: Erden T, Gültepe BS, Küçükdurmaz F. Periprosthetic joint infection with streptococcus dysgalactiae subspecies equisimilis: Case report. Jt Dis Relat Surg 2020;31(2):399-402.

\section{ABSTRACT}

Streptococcus dysgalactiae (SD) is a common pathogen among elderly population. However, to our knowledge, there is no periprosthetic joint infection case reported that is infected with Streptococcus dysgalactiae subspecies equisimilis (SDSE) in the English literature. In this article, we report a 77-year-old male patient who had undergone total knee arthroplasty three years ago and had the diagnosis of cellulitis at his leg followed by swelling, pain and hyperemia localized at his knee. Three knee aspirations were performed and the SDSE was identified. There was no direct contact of patient to animals.

Keywords: Arthroplasty, periprosthetic joint infection, Streptococcus dysgalactiae.

left leg. He had the diagnosis of cellulitis at his leg and used cefazoline for two weeks. The cellulitis was healed clinically but then he was consulted to our clinic with swelling and hyperemia localized at his knee which was performed total knee arthroplasty (TKA) three years ago. Pain, swelling, hyperemia and limited range of motion were observed in the left knee during physical examination. He had a history of consumption of unpasteurized milk. There was no direct contact to pets or any other animals. A written informed consent was obtained from the patient.

Three aspirations were performed. One aspiration was positive. The antibiogram demonstrated antibiotic sensitivity against trimethoprimsulfamethoxazole, ampicillin, linezolid, clindamycin, erythromycin, cefotaxime, ceftriaxone, vancomycin, levofloxacin and penicillin. Then, the final diagnosis was established based on the adaptation of the Musculoskeletal Infection Society (MSIS) criteria (Table I): ${ }^{[7]}$

1. One positive culture

2. High C-reactive protein (CRP) $(11.21 \mathrm{mg} / \mathrm{L})$ 


\section{TABLE I}

Musculoskeletal Infection Society criteria for periprosthetic joint infection

Major criteria

1. Two positive periprosthetic cultures with phenotypically identical organisms, or

2. A sinus tract communicating with the joint

Minor criteria

1. Elevated serum erythrocyte sedimentation rate (ESR) and serum C-reactive protein (CRP) concentration,

2. Elevated synovial leukocyte count,

3. Elevated synovial polymorphonuclear neutrophils (PMN\%),

4. Presence of purulence in the affected joint,

5. Isolation of a microorganism in one culture of periprosthetic tissue or fluid, or

6. Greater than five neutrophils per high-power field in five high-power fields observed from histologic analysis of periprosthetic tissue at 400 magnification.

Diagnosis can be established when one major criterion exits or four of the minor criteria exist.

\& erythrocyte sedimentation rate (ESR) $(57 \mathrm{~mm} / \mathrm{h})$

3. More than $90 \%$ polymorphonuclear neutrophils (PMN) in synovial fluid

4. $\quad$ Leukocyte esterase strip test showed $2+$

The SD was identified in the first sample (Figure 1). The aspiration sample was inoculated to aerobic and anaerobic blood culture tubes (Becton-Dickinson, New Jersey, USA). Also, some of the sample was reserved for gram staining and direct inoculation to blood agar (Salubris Inc. Pharmaceuticals, Istanbul,

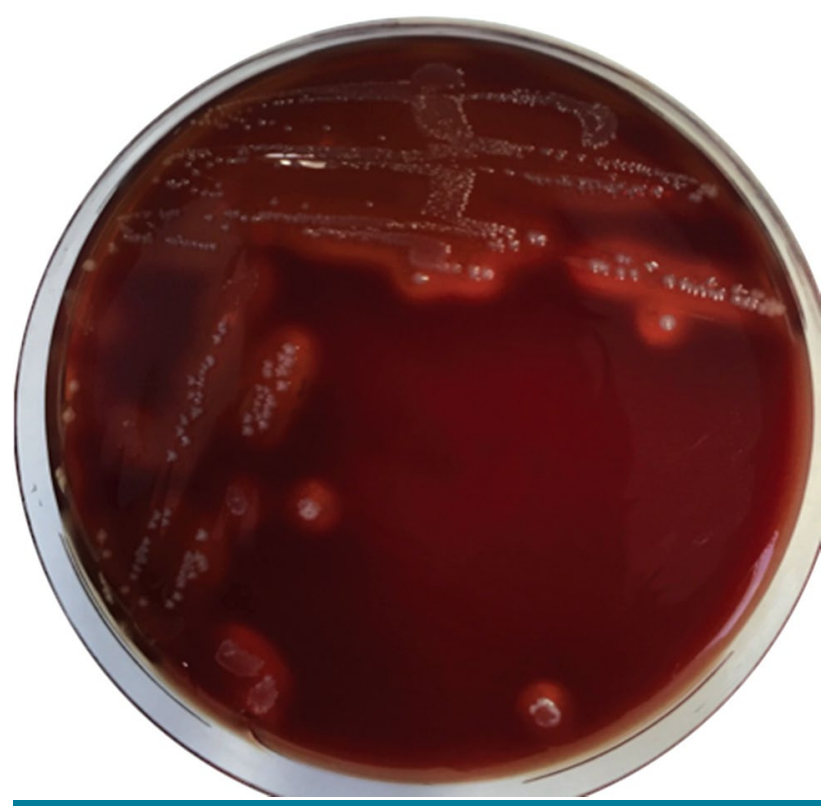

FIGURE 1. Patient had total knee arthroplasty history at his infected knee. Streptococcus dysgalactiae was determined by culture.
Turkey), eosin methylene blue agar (Salubris) and chocolate agar (Salubris). The samples were incubated in $37^{\circ} \mathrm{C}$ for $18-24$ hours in carbon dioxide medium. In gram staining, gram-positive cocci were seen. The blood culture tubes demonstrated signals for cultivation by day six. The cultivated bacteria were identified as SD using conventional and automatized systems (Vitek ${ }^{\circledast} 2$ Compact, BioMérieux, Craponne, France). The antibiogram for the identified species were determined using the Clinical and Laboratory Standards Institute (CLSI) criteria. ${ }^{[8]}$ For further identification and confirmation, Vitek ${ }^{\circledR}$ MS (MALDITOF) (BioMérieux) was used and the subspecies were identified as SDSE.

The case was considered as hematogenous spread of infection and as suggested in PJI consensus meeting, thorough debridement of the synovial sheath was performed and polyethylene insert was adapted. Postoperative antibiotic treatment was continued with third-generation cephalosporin for two weeks and then stopped when the infection symptoms were relieved, the CRP and the ESR levels dropped down and presented a plateau (Figure 2). The patient was followed-up for 11 months without recurrent infection.

\section{DISCUSSION}

The PJI is the most devastating complication of total joint arthroplasty. The incidence of PJI is around $1 \%$ in TKA and the most common agent is Staphylococcus aureus. Streptococci are responsible for 5 to $7 \%$ of deep infection cases after total joint arthroplasty. Most of the streptococci are group A, alpha or beta hemolytic type. According to our research, there is only one arthroplasty case infected with $\mathrm{SD}^{[6]}$ and this case is the only SDSE PJI reported in the literature. ${ }^{[9]}$ 


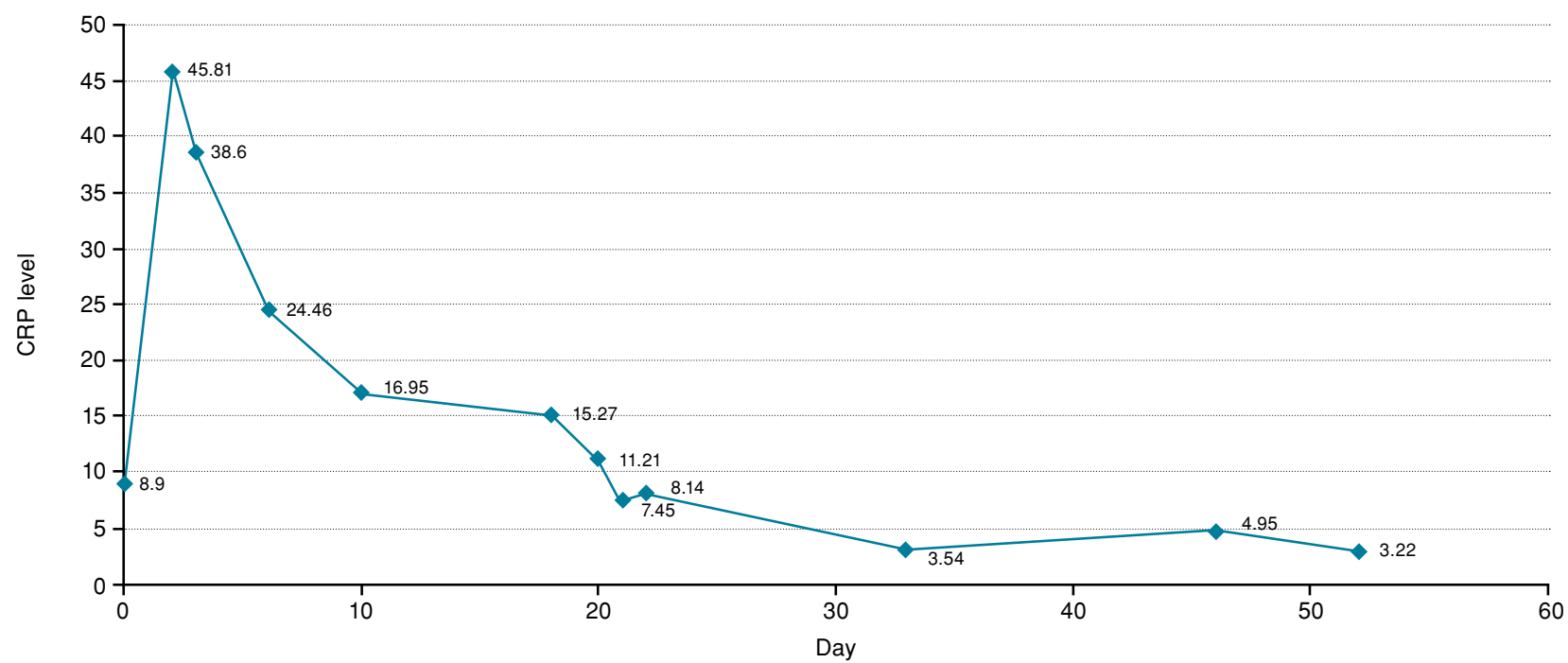

FIGURE 2. After surgical debridement and antibiotic treatment, CRP levels dropped down. After 30 days, CRP levels presented a plateau.

CRP: C-reactive protein.

Streptococcus dysgalactiae subspecies equisimilis has generally been recovered from the pharynges of carriers and from those with exudative pharyngitis and tonsillitis. There are a limited number of papers reporting SDSE infections in elderly population with an increasing incidence in this age group. ${ }^{[10]}$

Group G streptococcal cellulitis, septic arthritis at or near sites of parenteral injection, with bacteremia and subsequent hematogenous complications have been reported in intravenous (IV) drug users. In our case, the PJI was followed by a cellulitis at a near site. However, there was no history of IV drug use.

Streptococcus dysgalactiae subspecies equisimilis isolates remain almost uniformly susceptible to betalactam agents. To avoid delayed or poor responses of infections because of failure of penicillin/ cephalosporins, the addition of an aminoglycoside should be considered for serious infections. ${ }^{[1]}$ In patients with penicillin allergy, and in type I allergic patients, vancomycin and clindamycin is a sensible alternative. ${ }^{[11]}$

Although the group C streptococcus PJI is extremely rare, the incidence of infection is increasing in elderly population. The group C streptococci should be kept in mind as an infecting agent particularly in elderly patients.

\section{Declaration of conflicting interests}

The authors declared no conflicts of interest with respect to the authorship and/or publication of this article.

\section{Funding}

The authors received no financial support for the research and/or authorship of this article.

\section{REFERENCES}

1. Winn W Jr, Allen S, Janda W, Koneman E, Procop G, Schreckenberger P, editors. Koneman's Color Atlas and textbook of Diagnostic Microbiology. 6th ed. Philadelphia: Lippincott Williams and Wilkins; 2006.

2. Brandt CM, Spellerberg B. Human infections due to Streptococcus dysgalactiae subspecies equisimilis. Clin Infect Dis 2009;49:766-72.

3. Rider MA, McGregor JC. Group G streptococcus-an emerging cause of graft loss? Br J Plast Surg 1994;47:346-8.

4. Broyles LN, Van Beneden C, Beall B, Facklam R, Shewmaker PL, Malpiedi P, et al. Population-based study of invasive disease due to beta-hemolytic streptococci of groups other than A and B. Clin Infect Dis 2009;48:706-12.

5. Zaoutis T, Attia M, Gross R, Klein J. The role of group C and group $\mathrm{G}$ streptococci in acute pharyngitis in children. Clin Microbiol Infect 2004;10:37-40.

6. Park MJ, Eun IS, Jung CY, Ko YC, Kim YJ, Kim CK, et al. Streptococcus dysgalactiae subspecies dysgalactiae infection after total knee arthroplasty: a case report. Knee Surg Relat Res 2012;24:120-3.

7. Workgroup Convened by the Musculoskeletal Infection Society. New definition for periprosthetic joint infection. J Arthroplasty 2011;26:1136-8.

8. Clinical and Laboratory Standards Institute. Performance Standards for Antimicrobial Susceptibility Testing. 27th ed. CLSI document M100-S27; Clinical and Laboratory Standards Institute: Wayne, PA, USA; 2017. p. 84-90.

9. Atik OŞ. Which articles do we prefer to publish? Eklem Hastalik Cerrahisi 2018;29:1. 
10. Takahashi T, Sunaoshi K, Sunakawa K, Fujishima S, Watanabe $\mathrm{H}$, Ubukata $\mathrm{K}$, et al. Clinical aspects of invasive infections with Streptococcus dysgalactiae ssp. equisimilis in Japan: differences with respect to Streptococcus pyogenes and Streptococcus agalactiae infections. Clin Microbiol
Infect 2010;16:1097-103.

11. Rantala S. Streptococcus dysgalactiae subsp. equisimilis bacteremia: an emerging infection. Eur J Clin Microbiol Infect Dis 2014;33:1303-10. 